\title{
Motifome comparison between modern human, Neanderthal and Denisovan
}

\author{
Matyas F. Cserhati ${ }^{1 *}$ (D), Mary-Ellen Mooter ${ }^{2}$, Lauren Peterson ${ }^{3}$, Benjamin Wicks ${ }^{4}$, Peng Xiao ${ }^{1}$, Mark Pauley ${ }^{3 *}$ \\ and Chittibabu Guda ${ }^{1}$
}

\begin{abstract}
Background: The availability of the genomes of two archaic humans, Neanderthal and Denisovan, and that of modern humans provides researchers an opportunity to investigate genetic differences between these three subspecies on a genome-wide scale. Here we describe an algorithm that predicts statistically significant motifs based on the difference between a given motif's actual and expected distributions. The algorithm was previously applied to plants but was modified for this work.
\end{abstract}

Results: The result of applying the algorithm to the human, Neanderthal, and Denisovan genomes is a catalog of potential regulatory motifs in these three human subspecies. We examined the distributions of these motifs in genetic elements including human retroviruses, human accelerated regions, and human accelerated conserved noncoding sequences regions. Differences in these distributions could be the origin of differences in phenotype between the three subspecies. Twenty significant motifs common to all three genomes were found; thirty-three were found in endogenous retroviruses in Neanderthal and Denisovan. Ten of these motifs mapped to the $22 \mathrm{bp}$ core of MiR-1304. The core of this genetic element regulates the ENAM and AMTN genes, which take part in odontogenesis and whose 3' UTRs contained significant motifs. The introns of 20 genes were found to contain a large number of significant motifs, which were also overrepresented in 49 human accelerated regions. These genes include NAV2, SorCS2, TRAPPC9, GRID1, PRDM16, CAMTA1, and ASIC which are all involved in neuroregulation. Further analysis of these genes using the GO database indicates that many are associated with neurodevelopment. Also, varying numbers of significant motifs were found to occur in regions of the Neanderthal and Denisovan genomes that are missing from the human genome, suggesting further functional differences between modern and archaic humans.

Conclusion: Although Neanderthal and Denisovan are now extinct, detailed examination of elements from their genomes can shed light on possible phenotypic and cognitive differences between these two archaic human subspecies and modern humans. Genetic similarities and differences between these three subspecies and other fossil hominids would also be of interest.

Keywords: Human, Neanderthal, Denisovan, Genome, Promoter, UTR, Intron, Motifome

\section{Background}

The recent sequencing of the Neanderthal and Denisovan genomes has provided an exciting opportunity to unravel the genetic differences between modern humans and our two closest relatives [1-3]. Up until now, the

\footnotetext{
*Correspondence: cserhati@uthscsa.edu; mpauley@unomaha.edu 'Department of Genetics, Cell Biology and Anatomy, University of Nebraska Medical Center, College of Medicine, Omaha, NE 68198-5145, USA ${ }^{3}$ School of Interdisciplinary Informatics, University of Nebraska at Omaha, Omaha, NE 68182-0116, USA

Full list of author information is available at the end of the article
}

majority of analyses performed on the Neanderthal and Denisovan genomes have been restricted to the analysis of polymorphisms, population dynamics, and individual genes; little has been done with respect to analyzing genetic regulation. Since the two archaic hominin subspecies are extinct, such a study is made difficult by the fact that direct examination of gene activity is not possible. However, as the genomes of modern human, Neanderthal, and Denisovan (HND) are very similar to each other-some have theorized that modern humans, Neanderthals and Denisovans interbred [4] - gene activity can be inferred by 
examining changes in promoters and other regulatory regions which, in turn, would correspond to changes in transcription factor binding sites [5]. Thus, the presence or absence of motifs in the promoter regions of these subspecies could indicate biological, and therefore phenotypic, differences between them and could shed light on the molecular basis of human genetic variation [6-9].

Armed with the HND genomes, a detailed genomic analysis of these three subspecies can be performed. Here we are interested in differences in the motif content of genes. Indeed, several genes have been identified which exhibit variation between modern humans and Neanderthals. These include the ABO blood group locus, a taste receptor, as well as the gene MC1R which could code for red hair and light skin [10]. In addition, a number of genetic elements-e.g., human accelerated regions (HARs), human accelerated conserved noncoding sequences regions (HACNSs), and transposon elements, such as microRNAs (miRNA) elements and endogenous retroviruses-have been discovered that reflect functional differences between modern humans and the archaic hominins. HACNSs are important in that they are uniquely conserved sequences (thus indicating function) in human and contain cisregulatory transcriptional enhancers active during development; transposon and microRNA elements are important since about $40 \%$ of the human genome is made up of retrotransposons [11] and since microRNA elements regulate more than $30 \%$ of all protein-coding genes [12].

In this paper, we use an algorithm described in previous works $[13,14]$ to generate and rank a catalogue of all motifs in the whole genomes and several subgenomic regions in human, Neanderthal, and Denisovan. In part, the algorithm calculates the difference between a given motif's actual distribution and its expected distribution based on the base pair content of the genome. We then determine if the significant motifs, those for which the actual occurrence is higher than expected, have any biological significance by looking for them in the high-quality transcription factor binding profile JASPAR database [15] and determining whether they are present in any genetic elements (promoters, miRNAs, functionally conserved non-coding regions, etc.). The presence of these significant motifs could indicate biological differences between modern and archaic humans. Other researchers can use these motifs in their own research to help in the discovery of possible functional genetic elements and to further elucidate the genetic differences between modern and archaic humans.

\section{Methods}

\section{Sequence sets}

For human, the whole genome sequence was downloaded from the RepeatMasker website (SCR_012954) [16]: http://
www.repeatmasker.org/genomes/hg19/RepeatMaskerrm330-db20120124/hg19.fa.out.gz. The core, proximal, and distal promoter sets were downloaded from the Eukaryotic Promoter Database (EPD) (SCR_002485) (https://epd.vitalit.ch/seq_download.php) [17]. Core promoters were determined to be $300 \mathrm{bp}$ long, proximal promoters as $1000 \mathrm{bp}$ long, and distal promoters to be 3000 bp long. The EPD database is built on the hg19 genome assembly. The introns for human (build 37.1) were downloaded from http://bpg. utoledo.edu/ afedorov/lab/EID/hs37p1.EID.tar.gz [18], and the human $5^{\prime}$ and 3' UTR sets were downloaded from http://utrdb.ba.itb.cnr.it/home/download [19]. The human sequence sets were filtered with Repeat Masker [16].

Mouse was selected as an outlier species with which to compare the three hominin subspecies. We chose mouse because it is a well-tested mammalian system, and also has available the corresponding sequence sets with human. The mouse whole genome sequence was downloaded from the UCSC website (SCR 005780): http://hgdownload.cse.ucsc.edu/goldenPath/ mm10/bigZips/chromFaMasked.tar.gz. The promoter sequences as well as the $5^{\prime}$ and $3^{\prime}$ UTRs were also downloaded together with the human data sets. The mouse introns (build 37.1) were also downloaded from the EID database: http://bpg.utoledo.edu/ afedorov/lab/EID/mm37p1.EID.tar.gz.

The chimpanzee genome was downloaded from the UCSC website: http://hgdownload.cse.ucsc.edu/golden Path/panTro2/bigZips/chromFa.tar.gz.

The vcf files for the Neanderthal and Denisovan genome were downloaded from http://cdna.eva.mpg.de/ne andertal/altai/AltaiNeandertal/ and http://cdna.eva.mpg. de/denisovan/ and converted to fasta format by a python script. A database was made from these sequences and the human reference transcript was aligned to orthologous Denisovan transcripts to retrieve promoter and intron sequences.

The whole genome sequence of Neanderthal and Denisovan as well as the core, proximal, and distal promoter sets and the set of all introns of Denisovan are available at http://golgi.unmc.edu/HumanMotifomeData/.

The sequence for has-mir-1304 was retrieved from the miRBase database (SCR_003152) [20] at http://www.mir base.org/cgi-bin/get_seq.pl?acc=MI0006371. The chimp genome (panTro2) was downloaded from http:// hgdownload.cse.ucsc.edu/goldenPath/panTro2/bigZips/c hromFa.tar.gz. The annotation for human genes was retrieved from the GeneCards database (http://www.gen ecards.org/).

\section{Scoring and selection procedure of motifs}

The method for predicting and scoring motifs in a given sub-genomic set of sequences builds upon the methods 
of previous works $[13,14,21,22]$. The reader is referred to these papers for a detailed description of motif prediction and scoring. In this work, however, we have refined and improved the motif detection algorithm to provide more robust results.

First of all, the sequences used were all filtered for repeat sequences using the RepeatMasker software. Next, the motif scoring scheme was normalized. According to the new method, the motif score is now

$$
S=O b s /(\operatorname{Exp}+O b s)
$$

where $\mathrm{Obs}$ is the observed occurrence of a given motif within a given sub-genomic set of sequences, and Exp is the expected number of occurrences, given the base pair distribution (\%A, C, G, and T). This way, the score value will always be between 0 and 1 . A score of $S=0.5$ means that the motif occurs just as many times as it is expected to occur and is biologically meaningless. Higher scores (closer to 1.0) correspond to motifs which occur more times than expected, and thus correspond to biological relevance. Lower scores (closer to 0.0) correspond to motifs which occur less times than expected, and thus correspond to biological insignificance. This score value is calculated for all combinatorically possible motifs of a given length.

In the second step, the motifs of a given set are ranked in decreasing order according to their score value. For each motif length $(k=6 \ldots 10)$, and for each sub-genomic set of sequences, the average score value and the standard deviation are also calculated. A cutoff score value of $S_{\text {cut }}=S_{a v}+2 \cdot s t d e v$ is calculated. The reason 2 standard deviations are used is because this corresponds to a 5\% significance level, according to the normal distribution. Each motif with a score value above the cutoff value was taken to be significant.

In the next step, the same procedure was performed for a set of corresponding motifs from mouse. Mouse was used so as to filter out general mammalian motifs which are not specific to human, Denisovan, or Neanderthal. Thus, we would arrive at a set of significant human sequences and mouse sequences. The set of biologically significant sequences in human were then filtered with the significant mouse sequences. The number of significant motifs, their average score values and standard deviation values are provided in the Additional files 1, 2, 3 and 4 for human, Neanderthal, Denisovan and mouse. This way, for each motif length and each sub-genomic set of repeat-masked sequences, for each of the three hominin subspecies we were able to determine a set of normalized, filtered motifs for each of the subgenomic regions. The whole process can be seen in Fig. 1. Furthermore, the set of motifs were also validated in the next step by comparing them against the human position weight matrices (PWMs) from the JASPAR database.

The $p$-value for common motifs of lengths 6 to $10 \mathrm{bp}$ for different subgenomic regions between modern human and Denisovan was calculated in Excel using the hypergeometric distribution.

\section{JASPAR database validation}

Position frequency matrixes (PFMs) from the JASPAR database (SCR_003030) [15] and transformed into Position Weight Matrixes (PWMs) for human and mouse. Human PFMs were also used for Neanderthal and Denisovan (due to the similarity of the subspecies). Each motif from each sub-genomic sequence set and the whole genome for all motif lengths from 6 to 10 bp were matched against these PWMs, and the annotation for each such motif was noted. These annotations were marked for all scored motifs, which were ranked by decreasing score values. Each motif was marked with a 1 signifying the presence of at least one matching JASPAR motif and with a 0 if it didn't match anything. We applied a statistical test where we took the ranks of all matching motifs, and the ranks of all non-matching motifs, and ran a t-test comparing these values with each other. These $p$-values are available for all sequence sets and all motif lengths from 6 to 10 . In each and every case the $p$-value was statistically very significant $(p<1 \mathrm{e}-3)$.

\section{Motif search \\ Conversion of coordinates of genetic elements}

The conversion of the coordinates of the HAR elements (hg17) from Pollard et al. [23] and the conversion of the SNP/INDEL coordinates (hg18) from Zhang et al. [24] were performed at the UCSC site using the liftover utility (https://genome.ucsc.edu/cgi-bin/hgLiftOver) to translate these coordinates to hg19 coordinates.

\section{Other}

Transcript IDs were mapped to UniProt IDs at http:// www.uniprot.org/uploadlists/ (selecting From RefSeq Protein to UniProtKB) (SCR_002380). Gene Ontology Analysis was performed at the PANTHER website (SCR_004869) [25]: http://pantherdb.org/webservices/ go/overrep.jsp. Figures 2 and 3 were made in $\mathrm{R}$, and Figs. 4 and 5 were made using the Venn diagram software at http://bioinformatics.psb.ugent.be/webtools/ Venn/ . Chi-squared analysis for testing the statistical significance of the CG\% of the three subspecies in Table 2 was performed by using the chisq.test function in $\mathrm{R}$. 


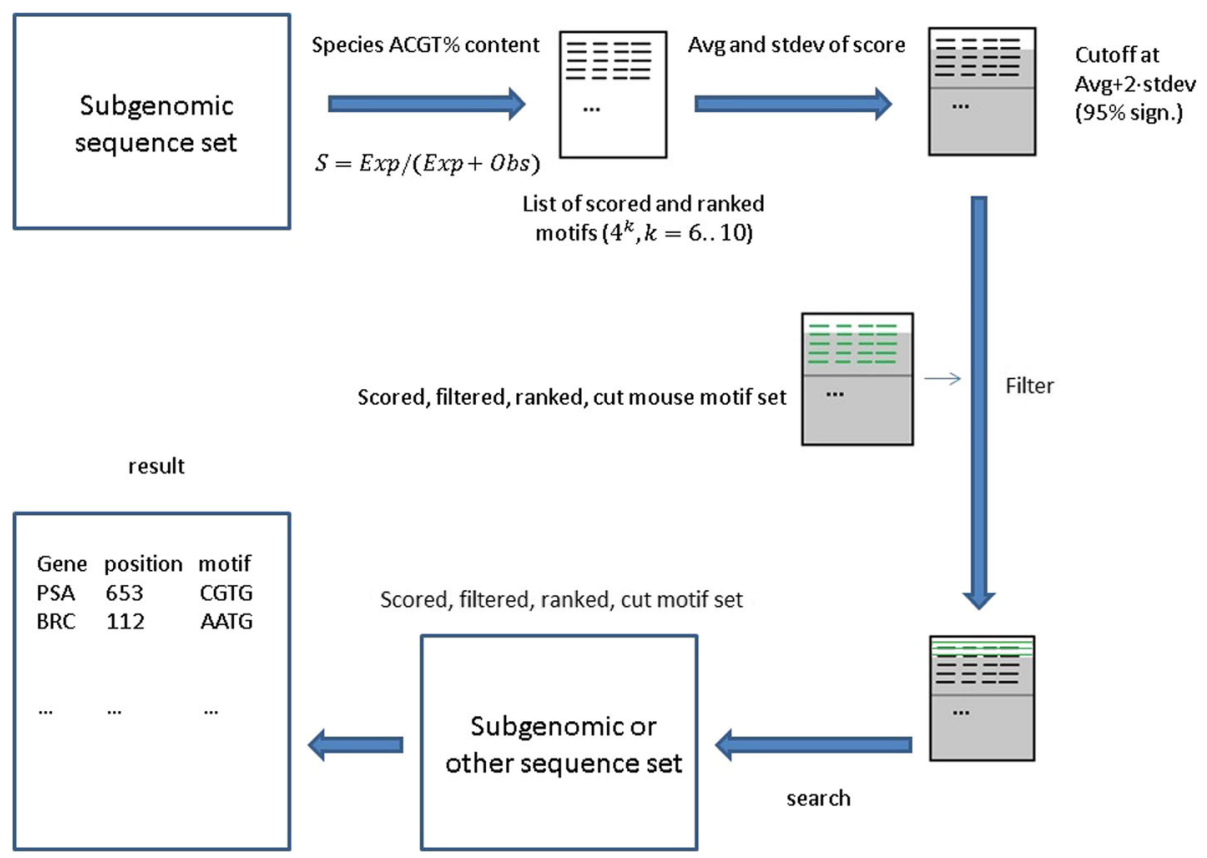

Fig. 1 Graphical overview of application of algorithm. First, the A, C, G, T\% for a given subgenomic sequence set is determined. Next, the statistics for each motif (for a given motif length from 6 to 10) is generated. This involves the calculation of the actual and expected occurrence for each motif as well as the score for each motif. Next, the set of motifs are filtered whose score is at least two standard deviations above the average score value. Next, the motif set is filtered again to remove general mammalian motifs. For this, the corresponding mouse motif set was used. Lastly, this set of statistically significant, double-filtered motif set can be used to search a given set of subgenomic or other sequence set

\section{Results}

\section{Principle of investigation}

In this paper, we use the term "motif" to mean a short stretch of DNA, 6-10 bp long, which overlaps the core of a transcription factor binding site; the motifome of an organism consists of all combinatorically possible motifs of all possible lengths, $6-10 \mathrm{bp}$ in this case. For example, there are $65,536\left(=4^{6}\right)$ possible hexamers in the hexamer motifome (all nucleic acid motifs of length six, ranging lexicographically from AAAAAA to TTTTTT). Assuming a random background nucleotide distribution, we would expect that motifs which occur in higher numbers than expected do so because they have experienced positive selective pressure due to the functionality they convey. Using the same reasoning, motifs which occur at the expected frequency or lower are considered biologically irrelevant [26]. The present work defines the motifomes of the three hominin subspecies-human, Neanderthal, and Denisovan-and predicts motifs which may take part in the regulation of gene sets that could cause phenotypic differences between the three subspecies.

Building on and refining the methodology of previous work $[13,14,22]$, we measured the statistical significance of the motif content of the whole genomes of human, Neanderthal, and Denisovan (as well as mouse for reference). Motifs in specific regions of the genome-core promoters, proximal promoters, distal promoters, all introns, 5' UTRs and 3' UTRs-were also determined so that these regions could be examine separately (see Table 1). Core, proximal, and distal promoters are defined as the segments of DNA 300, 1000 , and $3000 \mathrm{bp}$ upstream of the start codon of the coding sequence of a gene. The resulting set of statistically significant motifs was normalized by score and was double-filtered to remove general mammalian motifs (by filtering out those motifs, which also occurred in mouse) as well as low-scoring motifs, those whose actual occurrence is close its expected occurrence. For a detailed description of the algorithm, see $[13,14]$. An overview is given in Fig. 1, and is described in more detail in the Methods section. The motifs predicted by the algorithm as being statistically significant (that is, with a much higher occurrence than is expected) for all three subspecies and mouse can be found in the Additional files 1, 2, 3 and 4 .

\section{Motif comparison between human, Neanderthal, and Denisovan \\ Whole genome motifs}

Only the whole genome sequences of Neanderthal and Denisovan were available (see Methods), so we compared the 1000 highest-scoring significant whole genome motifs from modern human, Neanderthal, and Denisovan. 

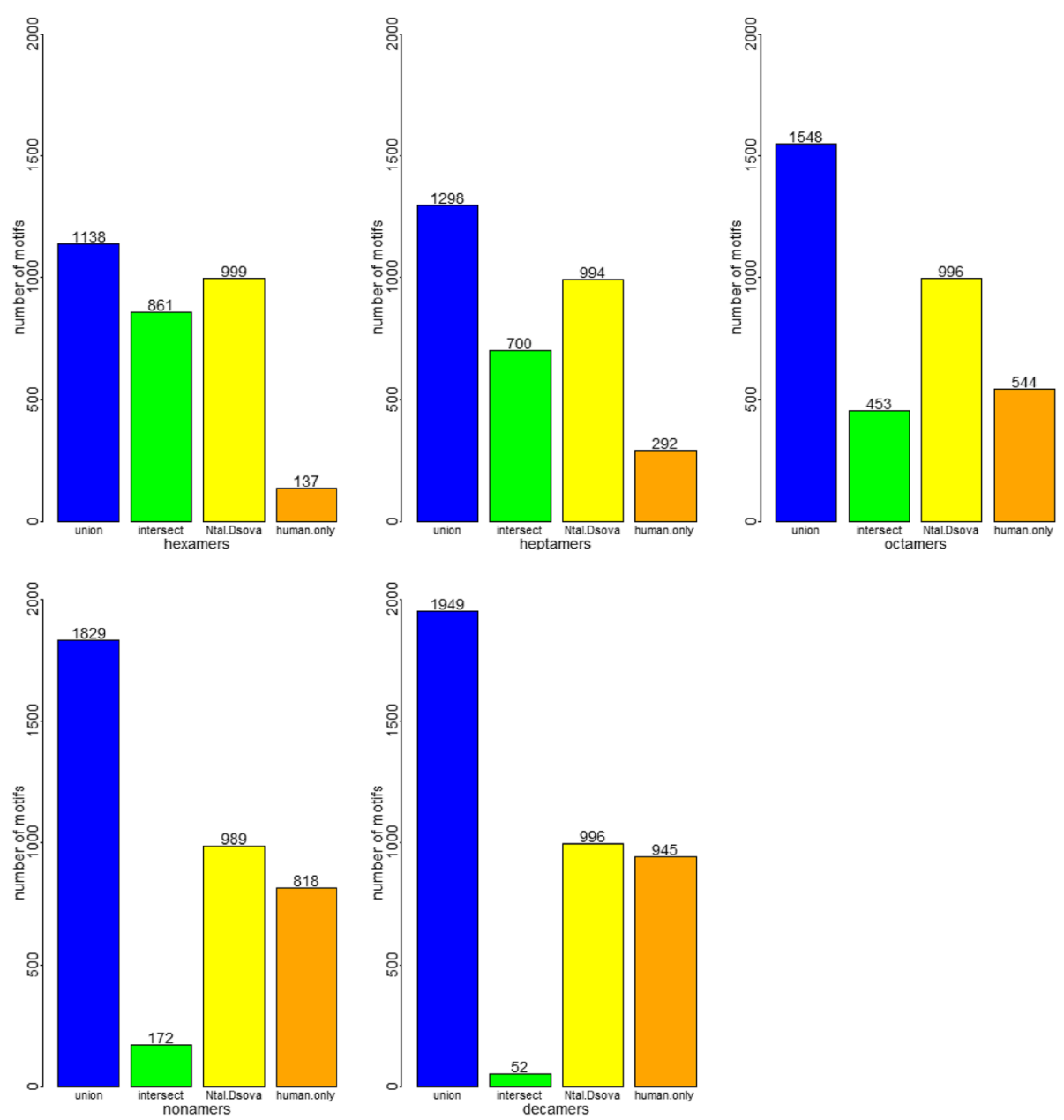

Fig. 2 Combination of top 1000 genome motifs from modern human, Neanderthal and Denisovan. The top 1000 genome motifs (hexamers to decamers) from modern human and the two archaic human species were compared with each other. What we can observe is that as the motif length increases, the intersect between all three species decreases, whereas the union of all motifs increases. The number of motifs common to both Neanderthal and Denisovan remain constantly very high, whereas the number of motifs unique to modern humans increases

The number of common motifs between different combinations of subspecies can be seen in Fig. 2. The general trend is that as the length of the motifs increases from six to ten, the union of significant motifs in all three subspecies increases, whereas the intersection between all three subspecies decreases. Furthermore, despite this trend, the number of significant motifs common to Neanderthal and Denisovan remains close to 1000 , whereas the number of significant humanspecific motifs increases steadily from 137 to 945 . This might indicate that the whole genome sequences of $\mathrm{Ne}$ anderthal and Denisovan are very similar (according to Table 2, their ACGT\% is almost identical), thus allowing for a greater turnover in motif content. The reason the number of common motifs with human decreases as the length of the motif increases is due to the fact that longer motifs are more specific sequentially, and their relative abundance is lower. A list of the top 20 significant motifs found in all three subspecies' genomes can be seen in Table 3 .

\section{Motif comparison between human and Denisovan in different sub-genomic regions}

Significant motif content was compared between human and Denisovan. The number of common motifs between modern human and Denisovan in the whole genome, the core, proximal, and distal promoters and all introns can be seen in Fig. 3. Here we can also see that with increasing motif length, the number of common motifs decreases. The decrease is steepest for the whole genome and for introns, and the least steep for core and proximal promoters. This means that even though there might be differences in the genome sequence between modern and archaic humans, the regulatory regions have few differences and have not diverged from each other. The top ten octamer motifs for core, proximal, and 

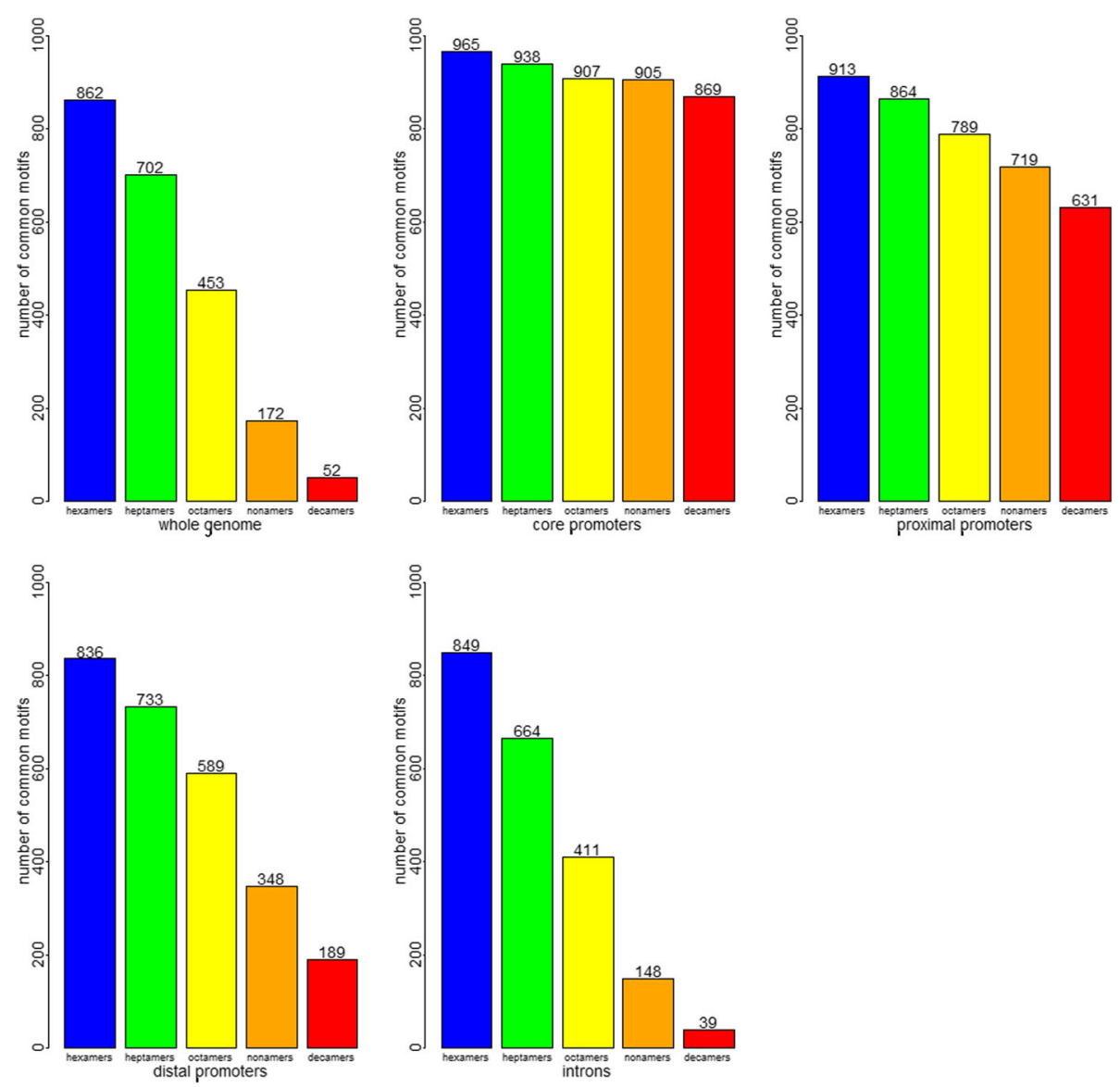

Fig. 3 Number of common motifs between modern human and Denisovan from different genomic regions. The top 1000 motifs coming from the whole genome, core, proximal, and distal promoters as well as all introns were compared between modern human and Denisovan for motif lengths 6-10. As we can see, as the length of the motif increases, the smaller number of common motifs. This is due to the fact that the longer the motif gets, the larger the possible number of motifs, thereby making it less likely that two species have the same motif. For core promoters, it is interesting to note that modern human and Denisovan have 869 decamer motifs in common (despite there being 1,048,576 possible decamers). P-values were calculated for each genomic sub-region and each motif length, and can be seen in Additional file 5 . All $p$-values were extremely statistically significant

distal promoters and all introns for human and Denisovan can be seen in Tables 4 and 5 . Information for $5^{\prime}$ and 3' UTRs are also provided for human.

\section{Experimentally verified motifs between human and Denisovan}

We searched the scientific literature for examples of genes common to human, Neanderthal, and Denisovan to see whether we could find motifs predicted by our algorithm in any element (e.g., promoters, introns, $5^{\prime}$ or 3' UTR) of these genes.

\section{Top motifs found in human and Denisovan}

Among the different sub-genomic regions in human and Denisovan we can see that the top 10 highest-ranking motifs in Tables 4 and 5 match to the well-known E2F1, EGR1, KLF5, SP1, SP2, and ZNF263 motifs.
We found that a variant of the KLF5 binding site (GCCCCGCC) occurs in the promoter of the KLF4 gene. KLF5 is a Krüppel-like transcription factor, which takes part in cell growth, proliferation, and differentiation. Whereas KLF4 inhibits cell growth by interacting with p21 and p53, KLF5 induces cell growth, and its increased mRNA levels can be found in active cells, such as the base of the crypt epithelium as well as the proliferative basal layer of the epidermis, where active cell division takes place in mice. This increase in KLF5 expression is due to the Egr1 protein, which itself is induced by MAPK [27]. Both KLF4 and KLF5 interact with the same cis-element, inhibit each other's activity, and they also exhibit tumor suppressor and oncogenic activities, respectively.

Frietze et al. [28] studied the distribution of 5000 ZNF263 (a zinc-finger transcription factor) binding sites within the human genome. They found 43 genes that 


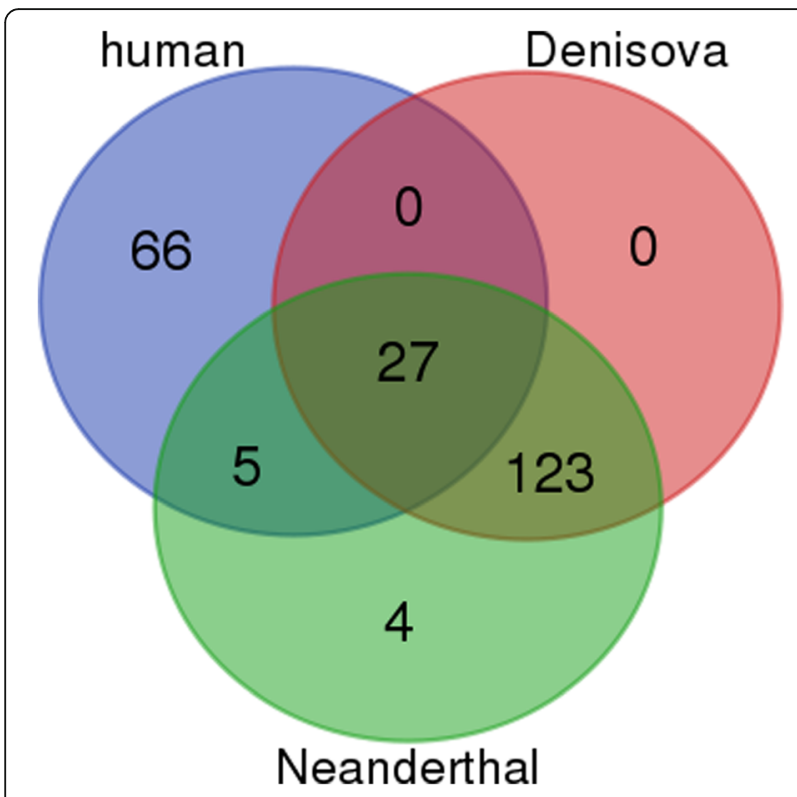

Fig. 4 Statistically significant intergenic motifs between modern and archaic humans. The Venn diagram shows the number of statistically significant whole genome motifs common to different combinations of modern human, Neanderthal and Denisovan found in the 26 intergenic HAR regions described by Pollard et al. (2006) [23]

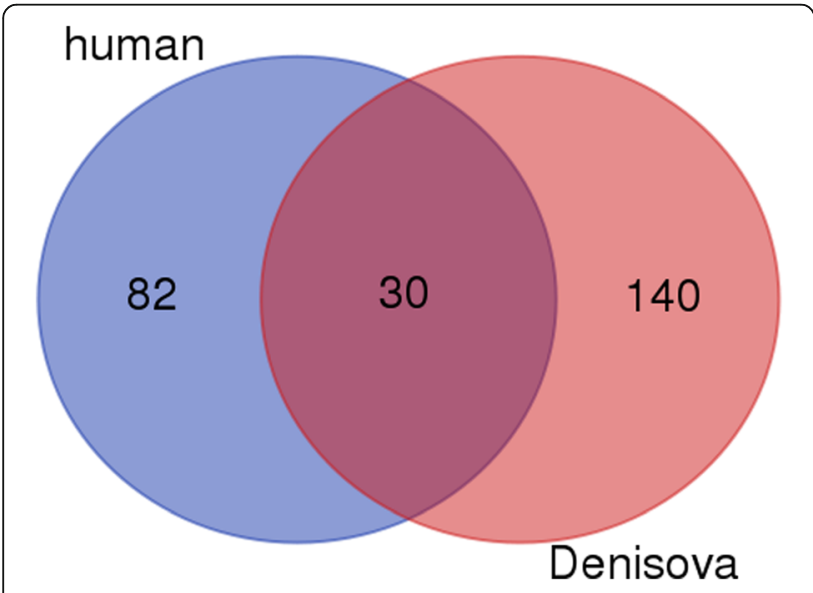

Fig. 5 Statistically significant intronic motifs between modern human and Denisovan. The number of statistically significant whole genome motifs common to different combinations of modern human and Denisovan found in the 20 intergenic HAR regions described by Pollard et al. (2006) [23]. The 82 motifs unique to modern humans were then searched for in the intron sequences of modern human. The top 189 transcripts which had the highest number of these motifs were then subsequently mapped to 135 UniProt IDs at the UniProt website, which in turn mapped to 261 specific gene names, for which GO analysis was performed
Table 1 Genomic regions analyzed in human, Denisovan and Neanderthal

\begin{tabular}{llll}
\hline Region & Modern human & Denisovan & Neanderthal \\
\hline Whole genome & $\sqrt{ }$ & $\sqrt{ }$ & $\sqrt{ }$ \\
Core promoters & $\sqrt{ }$ & $\sqrt{ }$ & $\times$ \\
Proximal promoters & $\sqrt{ }$ & $\sqrt{ }$ & $\times$ \\
Distal promoters & $\sqrt{ }$ & $\sqrt{ }$ & $\times$ \\
Introns & $\sqrt{ }$ & $\sqrt{ }$ & $\times$ \\
5' UTRs & $\sqrt{ }$ & $X$ & $\times$ \\
3' UTRs & $\sqrt{ }$ & $X$ & $\times$ \\
\hline
\end{tabular}

were up-regulated due to ZNF263 and 28 that were down-regulated. We found that the GGGAGGGG ciselement occurs in the promoters of 14 of these genes and that 11 were up-regulated and 3 were downregulated.

The Ras oncogene is a gene active for example in medullary thyroid cancer [29]. One gene activated by Ras is the calcitonin (CT) gene, the promoter of which contains a Ras-responsive transcriptional element (RREB) at position - 191 - -198 (CCCCCACC), which is a variant of the RREB found in the top ten elements found by our search. This demonstrates that the algorithm is capable of predicting motifs, which have already been experimentally verified.

\section{Neanderthal and Denisovan retroviruses in modern humans} So-called endogenous retroviruses (ERVs) make up 5-8\% of the human genome. Agoni et al. [30] reported 14 ERVs in the genome sequences of Neanderthal and/or Denisovan fossils. Such elements have also been identified in humans, which also cause disease [31]. Recently, Marchi et al. [32] discovered that eight of the HERVs previously discovered by Agoni et al. were also found in the human genome. These ERVs belong to the HERVK family of endogenous retroviruses, which have been active the most recently, and have seemingly infected the human lineage before modern humans split off Neanderthals and Denisovans. A list of whole genome motifs, which were found in these HERVs are provided in Table 6.

Table 2 ACGT\% values for the human, Neanderthal and Denisovan genomes

\begin{tabular}{lllll}
\hline Species & $\mathrm{A}$ & $\mathrm{T}$ & $\mathrm{C}$ & $\mathrm{G}$ \\
\hline Modern human & $29.78 \%$ & $29.82 \%$ & $20.19 \%$ & $20.21 \%$ \\
Neanderthal & $29.53 \%$ & $29.57 \%$ & $20.45 \%$ & $20.45 \%$ \\
Denisovan & $29.52 \%$ & $29.57 \%$ & $20.45 \%$ & $20.46 \%$ \\
Chi-square statistic & $1.17 \times 10^{-6}$ & & $4.98 \times 10^{-6}$ & \\
$p$-value & 1.0 & & 1.0 & \\
\hline
\end{tabular}


Table 3 Top 20 significant whole genome motifs found in the genomes of all three species

\begin{tabular}{|c|c|c|c|}
\hline Motif & Human score & Denisovan score & Neanderthal score \\
\hline ССТCCC & 0.818 & 0.87 & 0.87 \\
\hline GGGAGG & 0.817 & 0.869 & 0.869 \\
\hline CCCAGG & 0.825 & 0.854 & 0.854 \\
\hline CCTGGG & 0.825 & 0.854 & 0.854 \\
\hline CCCAGC & 0.797 & 0.859 & 0.859 \\
\hline GCTGGG & 0.797 & 0.859 & 0.859 \\
\hline CACACACACA & 0.978 & 0.996 & 0.996 \\
\hline CCAGGC & 0.787 & 0.848 & 0.847 \\
\hline CCCAGGC & 0.856 & 0.916 & 0.916 \\
\hline GCCTGG & 0.787 & 0.847 & 0.847 \\
\hline GCCTGGG & 0.856 & 0.915 & 0.915 \\
\hline ACACACACAC & 0.976 & 0.996 & 0.996 \\
\hline CCAGCC & 0.789 & 0.844 & 0.844 \\
\hline CACACACAC & 0.95 & 0.988 & 0.988 \\
\hline GGCTGG & 0.789 & 0.844 & 0.844 \\
\hline CCTGCC & 0.802 & 0.821 & 0.821 \\
\hline GGCAGG & 0.802 & 0.82 & 0.82 \\
\hline GTGTGTGTG & 0.95 & 0.988 & 0.988 \\
\hline AAAAAAAAA & 0.949 & 0.987 & 0.987 \\
\hline AAAAAAAA & 0.899 & 0.965 & 0.966 \\
\hline
\end{tabular}

The motif sequence and score are listed for modern human, Neanderthal and Denisovan

\section{Examination of motif content similarity in miR-1304}

Besides genes and ERVs, microRNA (miRNA) sequences were examined which were common between human and Denisovan. miRNAs are involved in the regulation of more than $30 \%$ of all human genes and take part in complex networks which regulate many cellular processes [12]. There are also a number of miRNAs, which are found only in present-day humans, and are therefore good candidates in discovering differences between modern and archaic humans such as Neanderthal and Denisovan [33]. For example, miR-1304 differs in only one single bp between human and Neanderthal, and is responsible for dental and other craniofacial differences [34].

Overall, ten significant whole genome motifs from human were found in the $22 \mathrm{bp}$ seed sequence of miR-1304 (CACATCTCACTGTAGCCTC[A/G]AA), which are listed in Table 6. MiR-1304 has also been shown to regulate two genes, ENAM and AMTN, which code for the enamelin and amelotin proteins, which take part in odontogenesis [35]. Statistically significant motifs were also found which occur in the 3' UTR of these genes (Table 7).

\section{Human accelerated regions}

Until now, many molecular genetic studies have focused on analyzing the coding sequences of genes, which are different between humans and other species. However, since many non-coding genetic elements make up the majority of functional elements in the genome, it would certainly be worthwhile to investigate these regions in order to identify such elements and to see what kinds of possible differences there are between human, Neanderthal and Denisovan $[6,23]$.

We found that the statistically significant decamer GCAGCCTTGG was found in intron 9 of the CENTG2 gene in both human (score $=0.867$ ) and Denisovan [7] This gene is responsible for differential limb development patterns. This intron contains a 546-bp region called HACNS1, or Human Accelerated Non-Coding Sequence 1, but is constrained in all but 16 humanspecific positions between human, chimp, rhesus, mouse, rat, dog, chicken, and frog. A shorter, 81-bp segment contains 13 of these $16 \mathrm{bp}$ differences. This 81 -bp long segment is identical in both human and Denisovan. The hexamer CAGCCT (score $=0.842$ ), and the nonamers GCAGCCTTG (score $=0.857$ ) and GGCACCCAC (score $=0.843$ ) were also among the top motifs that the algorithm found in Denisovan.

Also, Pollard et al. [23] identified 49 so-called Human Accelerated Regions (labelled HAR1-49) in the human genome that had substantial sequence differences compared to other animals. 94\% (46 of 49) of these are located in non-coding regions; 26 are found in intergenic regions, 20 in introns, 2 in coding regions, and 1 in RNA.

Each of the 26 intergenic regions has a BLAST hit with both the Neanderthal and the Denisovan genome, and the 20 intronic regions also have a BLAST hit with the Denisovan genome. Significant motifs from the human, Neanderthal, and Denisovan genomes and the human and Denisovan intronic regions are listed in Additional file 5, along with the HAR region that they map to.

Since a large number of base pair differences are present within these elements compared to other mammals (for example there is an 18 bp difference between human and chimpanzee in HAR1), it would be interesting to see how much of a difference exists between modern humans, Neanderthals, and Denisovans.

Between modern humans, Neanderthal and Denisovan, there are 27 whole genome motifs which map to these intergenic regions. There are 66 motifs unique to modern human, 4 to Neanderthal, and 127 which are unique to both Neanderthal and Denisovan. The number of motifs common to different combinations of subspecies can be seen in Fig. 4. The four whole genome motifs unique 
Table 4 Top 10 octamers from different sub-genomic regions in modern human

\begin{tabular}{|c|c|c|c|c|}
\hline Motif & Observed & Expected & Score & JASPAR annotation \\
\hline \multicolumn{5}{|l|}{ Core promoters } \\
\hline GGGGCGGG & 4545 & 19.014 & 0.996 & E2F1 \\
\hline GGGCGGGG & 4729 & 19.014 & 0.996 & \\
\hline CCCCGCCC & 4371 & 19.014 & 0.996 & EGR1|KLF5|SP1|SP2 \\
\hline GGCGGGGC & 3740 & 19.014 & 0.995 & \\
\hline GCCCCGCC & 3488 & 19.014 & 0.995 & EGR1|KLF5|SP1|SP2 \\
\hline ggCCCGCCCC & 4180 & 19.014 & 0.995 & EGR1|KLF5|SP1|SP2 \\
\hline GGGGGCGG & 2802 & 19.014 & 0.993 & CTCF \\
\hline CCGCCCCC & 2492 & 19.014 & 0.992 & EGR1|SP1|SP2 \\
\hline GGCCCCGC & 1988 & 19.014 & 0.991 & \\
\hline GCGGGGCC & 2055 & 19.014 & 0.991 & \\
\hline \multicolumn{5}{|c|}{ Proximal promoters } \\
\hline GGGCGGGG & 7145 & 53.738 & 0.993 & \\
\hline GGGGCGGG & 6893 & 53.738 & 0.992 & E2F1 \\
\hline CCCGCCCC & 6585 & 53.738 & 0.992 & EGR1|KLF5|SP1|SP2 \\
\hline CCCCGCCC & 6980 & 53.738 & 0.992 & EGR1|KLF5|SP1|SP2 \\
\hline GGCGGGGC & 5368 & 53.738 & 0.99 & \\
\hline GCCCCGCC & 5398 & 53.738 & 0.99 & EGR1|KLF5|SP1|SP2 \\
\hline GGGGGCGG & 4443 & 53.738 & 0.988 & CTCF \\
\hline CCGCCCCC & 4084 & 53.738 & 0.987 & EGR1|SP1|SP2 \\
\hline ССССТССС & 4863 & 79.277 & 0.984 & KLF5|SP1|SP2 \\
\hline GGGAGGGG & 4628 & 79.277 & 0.983 & ZNF263 \\
\hline \multicolumn{5}{|l|}{ Distal promoters } \\
\hline GGGCGGGG & 7145 & 53.738 & 0.993 & \\
\hline GGGGCGGG & 6893 & 53.738 & 0.992 & E2F1 \\
\hline CCCGCCCC & 6585 & 53.738 & 0.992 & EGR1|KLF5|SP1|SP2 \\
\hline CCCCGCCC & 6980 & 53.738 & 0.992 & EGR1|KLF5|SP1|SP2 \\
\hline GGCGGGGC & 5368 & 53.738 & 0.99 & \\
\hline GCCCCGCC & 5398 & 53.738 & 0.99 & EGR1|KLF5|SP1|SP2 \\
\hline GGGGGCGG & 4443 & 53.738 & 0.988 & CTCF \\
\hline CCGCCCCC & 4084 & 53.738 & 0.987 & EGR1|SP1|SP2 \\
\hline сСССТССС & 4863 & 79.277 & 0.984 & KLF5|SP1|SP2 \\
\hline GGGAGGGG & 4628 & 79.277 & 0.983 & ZNF263 \\
\hline \multicolumn{5}{|l|}{ Introns } \\
\hline GGGTGGGG & 62,500 & 3466.985 & 0.947 & \\
\hline GGGGTGGG & 59,103 & 3466.985 & 0.945 & \\
\hline GGGGAGGG & 56,341 & 3466.985 & 0.942 & ZNF263 \\
\hline GGGCTGGG & 55,491 & 3466.985 & 0.941 & \\
\hline GGGAGGGG & 54,920 & 3466.985 & 0.941 & ZNF263 \\
\hline CCCCACCC & 54,636 & 3466.985 & 0.94 & KLF5|RREB1 \\
\hline сССТСССС & 53,123 & 3466.985 & 0.939 & KLF5|SP1 \\
\hline сСССТССС & 53,180 & 3466.985 & 0.939 & KLF5|SP1|SP2 \\
\hline CCCACCCC & 51,857 & 3466.985 & 0.937 & KLF5|RREB1 \\
\hline GGGCAGGG & 50,200 & 3466.985 & 0.935 & \\
\hline
\end{tabular}


Table 4 Top 10 octamers from different sub-genomic regions in modern human (Continued)

\begin{tabular}{|c|c|c|c|c|}
\hline Motif & Observed & Expected & Score & JASPAR annotation \\
\hline \multicolumn{5}{|l|}{$5^{\prime}$ UTR } \\
\hline GGCGGCGG & 3004 & 21.007 & 0.993 & \\
\hline GCGGCGGC & 3089 & 21.007 & 0.993 & \\
\hline GCCGCCGC & 2332 & 21.007 & 0.991 & \\
\hline CGGCGGCG & 2405 & 21.007 & 0.991 & \\
\hline CCGCCGCC & 2370 & 21.007 & 0.991 & \\
\hline GGGGCGGG & 1846 & 21.007 & 0.989 & E2F1 \\
\hline GGGCGGGG & 1770 & 21.007 & 0.988 & \\
\hline CGCCGCCG & 1803 & 21.007 & 0.988 & \\
\hline CCCGCCCC & 1784 & 21.007 & 0.988 & EGR1|KLF5|SP1|SP2 \\
\hline CCCCGCCC & 1740 & 21.007 & 0.988 & EGR1|KLF5|SP1|SP2 \\
\hline \multicolumn{5}{|l|}{ 3' UTR } \\
\hline CCCCACCC & 4064 & 143.926 & 0.966 & KLF5|RREB1 \\
\hline CCCACCCC & 3940 & 143.926 & 0.965 & KLF5|RREB1 \\
\hline ССССТССС & 3774 & 143.926 & 0.963 & KLF5|SP1|SP2 \\
\hline СCСТCCCC & 3679 & 143.926 & 0.962 & KLF5|SP1 \\
\hline CCCTGCCC & 3555 & 143.926 & 0.961 & ESR1|ESR2|TFAP2A|TFAP2C \\
\hline CCCAGCCC & 3527 & 143.926 & 0.961 & \\
\hline GGGTGGGG & 3470 & 143.926 & 0.96 & \\
\hline GGGGTGGG & 3461 & 143.926 & 0.96 & \\
\hline GGGAGGGG & 3292 & 143.926 & 0.958 & ZNF263 \\
\hline GGGGAGGG & 3207 & 143.926 & 0.957 & ZNF263 \\
\hline
\end{tabular}

The motif, observed and expected occurrence, the motif score as well as any corresponding annotation from the JASPAR database are all listed for the top 10 motifs from the core, proximal, and distal promoters as well as all introns, and 5' and 3' UTRs

to Neanderthal are CTTTGGGA, AGAAAATGTG, AAGTGCTG and ACAGGCTCTG.

Between humans and Denisovans there are 82 motifs which are unique to human introns. These motifs can be seen in Additional file 6. The number of motifs unique to modern human or Denisovan, and which are common to both can be seen in Fig. 5. We searched the human intron sequence set for these specific human-specific motifs, to see what kind of genes they fall in. The top 20 genes which have at least 77 unique motifs are listed in Table 8 along with their gene name and function.

Furthermore, beyond the top 20 genes, we took the top 189 transcript IDs which had the highest number of motifs (at least 71 unique motifs) coming from the 82 unique human intron motifs examined previously. These 189 transcripts subsequently mapped to 135 UniProt IDs at the UniProt website, which in turn mapped to 261 specific gene names. GO term analysis was done with these 261 genes $(p<0.05)$ at the Panther Database website, the result of which can be seen in Tables 9 and 10. The results of the GO term analysis are also available in Additional file 7.

\section{Conserved deleted regions in human}

McLean et al. [36] studied 583 so-called hCONDEL sequences in chimpanzee and human. These are called hCONDELs because they appear in highly conserved intergenic regions of the genome, and are present in chimpanzee, yet missing in human. Five hundred ten of these regions were validated experimentally by single reads, which span both sides of the region in the human genome. These hCONDEL regions have a median size of 2804 bp and show a skew towards GC-poor regions. These regions also fall close to genes, which take part in steroid hormone receptor signaling. Since these regions fall within intergenic regions, they might contain regulatory elements in the chimpanzee genome, which might cause phenotypic differences as compared to the human genomes.

We thought that it would be interesting to see whether these hCONDEL regions were also fully missing from the Neanderthal and Denisovan genomes. If some of them are also present in these two genomes, they might shed light on to possible differences between modern and archaic humans. 
Table 5 Top 10 octamers from different genomic sub-regions in Denisovan

\begin{tabular}{ccccc}
\hline Motif & Observed & Expected & Score & JASPAR annotation \\
\hline Core promoters & & & & \\
GGGGCGGG & 17,407 & 82.475 & 0.995 & EGR1|KLF5|SP1|SP2 \\
GGGCGGGG & 17,780 & 82.475 & 0.995 & \\
CCCGCCCC & 16,718 & 82.475 & 0.995 & \\
CCCCGCCC & 17,676 & 82.475 & 0.995 & \\
GGCGGGGC & 13,917 & 82.475 & 0.994 & EGR1|KLF5|SP1|SP2 \\
GCCCCGCC & 13,714 & 82.475 & 0.994 & \\
GGGGGCGG & 10,283 & 82.475 & 0.992 & \\
CCGCCCCC & 9785 & 82.475 & 0.992 & \\
GGCGGCGG & 8520 & 82.475 & 0.99 & \\
GCGGGGCG & 7806 & 82.475 & 0.99 & EGR1|SP1|SP2|TFAP2C
\end{tabular}

Proximal promoters

\begin{tabular}{|c|c|c|c|c|}
\hline GGGCGGGG & 26,905 & 280.231 & 0.99 & \\
\hline CCCCGCCC & 27,739 & 280.231 & 0.99 & EGR1|KLF5|SP1|SP2 \\
\hline GGGGCGGG & 25,911 & 280.231 & 0.989 & E2F1 \\
\hline CCCGCCCC & 26,181 & 280.231 & 0.989 & EGR1|KLF5|SP1|SP2 \\
\hline GCCCCGCC & 20,826 & 280.231 & 0.987 & EGR1|KLF5|SP1|SP2 \\
\hline GGCGGGGC & 19,887 & 280.231 & 0.986 & \\
\hline GGGGGCGG & 16,398 & 280.231 & 0.983 & CTCF \\
\hline CCGCCCCC & 15,932 & 280.231 & 0.983 & EGR1|SP1|SP2 \\
\hline GGCGGCGG & 14,897 & 280.231 & 0.982 & \\
\hline GCGGCGGC & 14,477 & 280.231 & 0.981 & \\
\hline \multicolumn{5}{|l|}{ istal promoters } \\
\hline CAGGCTGG & 93,573 & 1749.587 & 0.982 & \\
\hline CCAGGCTG & 89,626 & 1749.587 & 0.981 & \\
\hline CCAGCCTG & 88,343 & 1749.587 & 0.981 & \\
\hline CAGCCTGG & 85,501 & 1749.587 & 0.98 & \\
\hline GCCTCCCA & 78,433 & 1749.587 & 0.978 & \\
\hline GGGCGGGG & 35,288 & 844.888 & 0.977 & \\
\hline CCCCGCCC & 36,139 & 844.888 & 0.977 & EGR1|KLF5|SP1|SP2 \\
\hline CAGCCTCC & 75,312 & 1749.587 & 0.977 & \\
\hline GGAGGCTG & 71,186 & 1749.587 & 0.976 & \\
\hline GCCTGGGC & 48,884 & 1215.815 & 0.976 & NFYB \\
\hline \multicolumn{5}{|l|}{ trons } \\
\hline CAGGCTGG & $3,328,904$ & $83,571.71$ & 0.976 & \\
\hline CCAGGCTG & $3,208,495$ & $83,571.71$ & 0.975 & \\
\hline CCAGCCTG & $3,097,321$ & $83,571.71$ & 0.974 & \\
\hline CAGCCTGG & $2,984,998$ & $83,571.71$ & 0.973 & \\
\hline GCCTCCCA & $2,849,031$ & $83,571.71$ & 0.972 & \\
\hline ताताताT & $23,869,680$ & $742,109.9$ & 0.97 & \\
\hline TGGGAGGC & $2,675,429$ & $83,571.71$ & 0.97 & TAL1::GATA1 \\
\hline
\end{tabular}

Table 5 Top 10 octamers from different genomic sub-regions in Denisovan (Continued)

\begin{tabular}{lllll}
\hline Motif & Observed & Expected & Score & JASPAR annotation \\
\hline CAGCCTCC & $2,724,864$ & $83,571.71$ & 0.97 & \\
GGAGGCTG & $2,573,566$ & $83,571.71$ & 0.969 & \\
CCTCAGCC & $2,528,019$ & $83,571.71$ & 0.968 &
\end{tabular}

The motif, observed and expected occurrence, the motif score as well as any corresponding annotation from the JASPAR database are all listed for the top 10 motifs from the core, proximal, and distal promoters as well as all introns

Table 6 List of significant whole genome motifs found in Neanderthal and Denisovan ERVs

\begin{tabular}{|c|c|c|}
\hline Motif & HERV id(s) & Score \\
\hline AGGTGGGA & HERV-K-De1, 3, 6 & 0.855 \\
\hline CACACCTG & HERV-K-De1, 3, 5, 6 & 0.889 \\
\hline CAGGTGTG & HERV-K-De2 & 0.888 \\
\hline GGAGGGGC & HERV-K-De2 & 0.845 \\
\hline AAAAGAAA & HERV-K-De5, 7 & 0.862 \\
\hline AAAGAAAA & HERV-K-De5 & 0.864 \\
\hline ТTCTTTCT & HERV-K-De6 & 0.839 \\
\hline CACACCTGT & HERV-K-De1, 3, 5, 6 & 0.937 \\
\hline ACAGGTGTG & HERV-K-De2 & 0.937 \\
\hline CAGGTGTGG & HERV-K-De2 & 0.916 \\
\hline GTGGAGGGG & HERV-K-De2 & 0.838 \\
\hline AAGAAAAAG & HERV-K-De5 & 0.838 \\
\hline AAAAGAAAG & HERV-K-De7 & 0.848 \\
\hline AAAGAAAGA & HERV-K-De7 & 0.897 \\
\hline AGAAAAAGA & HERV-K-De5 & 0.85 \\
\hline AGAAAGAGA & HERV-K-De7 & 0.834 \\
\hline GAAAAGAAA & HERV-K-De5, 7 & 0.869 \\
\hline ACACACCTGT & HERV-K-De1, 3, 5, 6 & 0.873 \\
\hline CACACCTGTG & HERV-K-De1, 3, 5, 6 & 0.858 \\
\hline CTTTTCCCCA & HERV-K-De1, 3, 5, 6 & 0.854 \\
\hline CACAGGTGTG & HERV-K-De2 & 0.853 \\
\hline GGTGTGGAGG & HERV-K-De2 & 0.86 \\
\hline GTGGAGGGGC & HERV-K-De2 & 0.849 \\
\hline TGGGGAAAAG & HERV-K-De2, 7 & 0.852 \\
\hline AAAGAAAGAG & HERV-K-De7 & 0.882 \\
\hline AAGAAAGAGA & HERV-K-De7 & 0.879 \\
\hline AGGTGGGA & HERV-K-Ne1 & 0.855 \\
\hline CACACCTG & HERV-K-Ne1, 2 & 0.889 \\
\hline TTCTाTCT & HERV-K-Ne1 & 0.837 \\
\hline CACACCTGT & HERV-K-Ne1, 2 & 0.937 \\
\hline ACACACCTGT & HERV-K-Ne1, 2 & 0.873 \\
\hline CACACCTGTG & HERV-K-Ne1, 2 & 0.858 \\
\hline CTITTCCCCA & HERV-K-Ne1, 2 & 0.854 \\
\hline
\end{tabular}

The motif sequence, and the id of the HERV sequence that the motif was found in is given, as well as the motif score 
Table 7 Whole genome motifs in miR-1304 and 5' and $3^{\prime}$ UTR of the ENAM and AMTN genes

\begin{tabular}{lll}
\hline Motif & Target & Score \\
\hline Human & & \\
CCCTGC & ENAM 3' UTR & 0.845 \\
TCCCTGC & ENAM 3' UTR & 0.836 \\
TITCCTIT & ENAM 3' UTR & 0.819 \\
GCTGCC & AMTN 3' UTR & 0.816 \\
GCTGCCT & AMTN 3' UTR & 0.835 \\
Neanderthal & & \\
ACTGTAGCCT & miR-1304 Seed & 0.887 \\
CACTGTAGCC & miR-1304 seed & 0.85 \\
AAAAAA & ENAM 3' UTR & 0.84 \\
CCTGCC & ENAM 3' UTR & 0.821 \\
CCCTGCC & ENAM 3' UTR & 0.837 \\
CCTGCCT & ENAM 3' UTR & 0.891 \\
CTGCCTC & ENAM 3' UTR & 0.898 \\
AATCACTTG & ENAM 3' UTR & 0.846 \\
CCTGCCTCG & ENAM 3' UTR & 0.92 \\
TITCCTIT & ENAM 3' UTR & 0.866 \\
TIITT & AMTN 3' UTR & 0.84 \\
CTGCCTC & AMTN 3' UTR & 0.898 \\
\hline
\end{tabular}

Several statistically significant whole genome motifs from modern human and Neanderthal were found in the seed section of the miR-1304 miRNA, as well as the $3^{\prime}$ and $5^{\prime}$ UTR of the ENAM and AMTN genes

Therefore, after having extracted the hCONDEL regions from the Pan troglodytes genome, version 2, we blasted them against the Neanderthal and Denisovan genomes. Of the 583 hCONDEL regions, 287 (49.2\%) of them had a significant hit at least $50 \mathrm{bp}$ long, with at least $90 \%$ sequence identity.

We then looked to see how many significant genomic Denisovan and Neanderthal motifs (lengths 6 to 10) fall into these 287 dCONDEL and nCONDEL regions. These numbers are summed up in Table 11. As we can see, there is quite a large overlap between Neanderthal and Denisovan. A list of these motifs for both Neanderthal and Denisovan for motif lengths 6 to 10 can be found in Additional file 8 .

\section{Discussion}

We have performed the motif content analysis of the human, Neanderthal and Denisovan genome. With this analysis, we provide a catalogue of motifs and their motif score in seven and five sub-genomic regions in the human and Denisovan genome as well as the Neanderthal whole genome. This data is now available for other researchers to use and analyze further.

One of the main questions in this analysis is whether our predicted motifs have actual biological relevance. As we can see, for the three different types of promoter sets, the intron sets, and the $5^{\prime}$ and 3 ' UTR sequence sets, the highest-ranking motifs matched experimentally verified motifs which had already been described in the JASPAR database. Furthermore, they did so in a highly non-random manner. When comparing the predicted motifs based on their match with experimentally verified motifs, the experimentally verified ones have higher ranks, according to $p$-value measurements.

However, as a further test we wanted to see if the statistically significant motifs that we predicted fall within biologically active sites within the genome. We found that a number of our candidate motifs fall within the sequence of a number of HERV sequences, and the miRNA sequence miR-1304 and also fall within the 3' UTRs of a couple of genes which are regulated by this latter miRNA.

Another interesting area of validation was comparing regions which were either different in sequence between human and other species, or were missing from humans compared to Denisovan and Neanderthal. These were the HAR regions as well as the hCONDEL regions.

The study of over-represented statistically significant genome motifs in the 49 HAR regions [23] also validated the biological validity of our predicted motifs. Some of the top 20 genes found in this search are for example, neuron navigator 2 isoform 3 (NAV2), which is required for all-trans retinoic acid to induce neurite outgrowth in human neuroblastoma cells [37]. Another gene is SorCS2, which is a VPS10-domain family receptor, which takes part in protein trafficking, intracellular and intercellular signaling [38]. SorCS2 itself is expressed in the hippocampus and is also involved in synapse formation and neuron function [39]. Another gene, TRAPPC9 (trafficking protein particle complex 9) is highly expressed in the postmitotic neurons of the cerebral cortex, and mutations in this gene show defects in axonal connectivity [40]. GRID1, which encodes the glutamate D1 receptor, which is a member of the $\delta$-family of ionotropic glutamate receptors, acts like an adhesion molecule by linking the postsynaptic and presynaptic compartments [41]. Yet another gene, PRDM16 is responsible for regulating the amount of mitochondrial reactive oxygen species (mtROS), which is necessary for the development of neurons [42]. The deletion of CAMTA1 causes cerebellar atrophy and Purkinje cell degeneration in mice [43]. The acid-sensing ion channels (ASICs) form mechanoreceptors in the periphery, and localize to dorsal and lumbar root ganglia [44]. It is highly interesting that a number of genes with high motif content were found in this search, since Pollard et al. [23] found that $24 \%$ of the HAR regions they described were adjacent to neurodevelopmental genes. 
Table 8 Top 20 human genes with highest number of human-specific intronic motifs from analysis of HAR regions

\begin{tabular}{|c|c|c|c|}
\hline Refseq ID & Gene name & Function & Number of motifs \\
\hline NP_955533.1 & PRDM16 & PR domain containing 16 isoform 2 & 81 \\
\hline NP_689957.3 & SDK1 & protein sidekick-1 isoform 1 & 81 \\
\hline NP_570858.2 & PTPRN2 & receptor-type tyrosine-protein phosphatase N2 isoform 3 & 81 \\
\hline NP_570857.2 & PTPRN2 & receptor-type tyrosine-protein phosphatase N2 isoform 2 & 81 \\
\hline NP_115821.2 & MEGF11 & multiple epidermal growth factor-like domains protein 11 precursor & 80 \\
\hline NP_113654.3 & TRAPPC9 & trafficking protein particle complex 9 & 80 \\
\hline NP_071407.4 & $\mathrm{CDH} 23$ & cadherin-23 isoform 1 precursor & 79 \\
\hline NP_071397.2 & PRDM16 & PR domain containing 16 isoform 1 & 79 \\
\hline NP_065828.2 & SORCS2 & VPS10 domain-containing receptor SorCS2 precursor & 79 \\
\hline NP_060021.1 & GRID1 & glutamate receptor ionotropic, delta-1 precursor & 78 \\
\hline NP_056030.1 & CAMTA1 & calmodulin-binding transcription activator 1 isoform 1 & 78 \\
\hline NP_055729.2 & AGAP1 & arf-GAP with GTPase, ANK repeat and PH domain-containing protein 1 isoform 2 & 77 \\
\hline NP_003362.2 & VAV2 & guanine nucleotide exchange factor VAV2 isoform 2 & 77 \\
\hline NP_002838.2 & PTPRN2 & receptor-type tyrosine-protein phosphatase N2 isoform 1 precursor & 77 \\
\hline NP_001785.2 & $\mathrm{CDH} 4$ & cadherin-4 isoform 1 preproprotein & 77 \\
\hline NP_001127870.1 & VAV2 & guanine nucleotide exchange factor VAV2 isoform 1 & 77 \\
\hline NP_001104488.1 & NAV2 & neuron navigator 2 isoform 3 & 77 \\
\hline NP_001085.2 & ASIC2 & acid-sensing ion channel 2 isoform MDEG1 & 77 \\
\hline NP_001076044.1 & RBFOX3 & RNA binding protein fox-1 homolog 3 & 77 \\
\hline NP_001032208.1 & AGAP1 & arf-GAP with GTPase, ANK repeat and PH domain-containing protein 1 isoform 1 & 77 \\
\hline
\end{tabular}

Statistically significant intron motifs were found in 20 intronic HARs between modern human and Denisovan. Eighty-two of them were shown to be specific to modern human. These are the top 20 genes with the highest number of these human-specific intron motifs (at least 77 of them) in their introns. Listed are the genes' Refseq ID, their gene name, function and the number of motifs their introns contain

This validates the fact that the motifs that we found are indeed biologically relevant and meaningful.

As we can see, five of the top 32 biological function GO terms, and five of the top 15 cellular component GO terms are involved in neurogenesis and neuron development, which are indicative of the differential neurological functions between modern and archaic humans. Not only that, but GO terms for ion channel complexes and transmembrane transporter complexes were also found. No significant GO terms for molecular processes were retrieved.

Analyzing hCONDEL regions also produced interesting results. These regions are specifically missing from the human genome, and as such gave us an opportunity to analyze the motif content difference between Neanderthal and Denisovan. Overall, it seems that the whole genome sequence similarity between these two archaic human subspecies is very high, since they both contained the same 287 hCONDEL regions, which are missing from human.

Overall, 44 statistically significant whole genome motifs mapped to these 287 regions, which differ between Neanderthal and Denisovan. Of these, among the motifs which only occur in Denisovan, the motif TGCCCAGACT $\quad($ score $=0.862$ ) corresponds to the
P63 Responsive Element [45]. P63, a member of the TP53 family of proteins is involved in certain types of tumors, such as vulvar cancer. Its expression is negatively correlated by miR-223-5p [46]. Among the 64 statistically significant whole genome motifs, which differ between Neanderthal and Denisovan, and which only occur in Neanderthal, the nonamer AGAGGGAG corresponds to the SP2 motif, and the CCAGGCCT motif corresponds to the TP63 motif identified earlier.

\section{Conclusions}

In summary, we have seen that despite Neanderthal and Denisovan having gone extinct, we are still able to discern certain genetic elements, which shed light onto the possible phenotypic differences between the two archaic human subspecies as well as modern human. Indeed, it would also be highly interesting to see what similarities and differences are there between these three subspecies and other fossil hominids.

In Table 2 we can see that the CG\% between Neanderthal and Denisovan are almost identical (at most there is a $0.01 \%$ difference), whereas the CG\% between modern human and the two archaic human subspecies is $0.25-0.26 \%$. However, the variation of 
Table 9 Biological processes found through GO term analysis

\begin{tabular}{|c|c|c|c|c|c|}
\hline GO term & Genes in database & Genes found & Expected & Fold enrichment & $P$-value \\
\hline Single-organism developmental process & 5316 & 61 & 27.63 & 2.21 & 8.94E-08 \\
\hline Developmental process & 5402 & 61 & 28.08 & 2.17 & 1.84E-07 \\
\hline System development & 4138 & 52 & 21.51 & 2.42 & 3.96E-07 \\
\hline Single-multicellular organism process & 5509 & 61 & 28.63 & 2.13 & 4.41E-07 \\
\hline Anatomical structure development & 5059 & 58 & 26.29 & 2.21 & 5.19E-07 \\
\hline Cell adhesion & 1103 & 25 & 5.73 & 4.36 & 3.16E-06 \\
\hline Biological adhesion & 1108 & 25 & 5.76 & 4.34 & 3.47E-06 \\
\hline Multicellular organism development & 4733 & 54 & 24.6 & 2.2 & $5.41 \mathrm{E}-06$ \\
\hline Single-organism process & 12,622 & 94 & 65.6 & 1.43 & 2.04E-05 \\
\hline Multicellular organismal process & 6584 & 64 & 34.22 & 1.87 & $3.08 \mathrm{E}-05$ \\
\hline Nervous system development & 2200 & 33 & 11.43 & 2.89 & 1.04E-04 \\
\hline Cell-cell adhesion & 680 & 18 & 3.53 & 5.09 & 1.27E-04 \\
\hline Cellular developmental process & 3501 & 42 & 18.2 & 2.31 & $3.60 \mathrm{E}-04$ \\
\hline Cell differentiation & 3425 & 41 & 17.8 & 2.3 & $6.09 \mathrm{E}-04$ \\
\hline Regulation of multicellular organismal process & 2656 & 35 & 13.8 & 2.54 & $8.50 \mathrm{E}-04$ \\
\hline Homophilic cell adhesion via plasma membrane adhesion molecules & 155 & 9 & 0.81 & 11.17 & $1.18 \mathrm{E}-03$ \\
\hline Generation of neurons & 1391 & 24 & 7.23 & 3.32 & 1.37E-03 \\
\hline Cell-cell adhesion via plasma-membrane adhesion molecules & 212 & 10 & 1.1 & 9.08 & 1.57E-03 \\
\hline Regulation of cellular component organization & 2292 & 31 & 11.91 & 2.6 & 3.40E-03 \\
\hline Neurogenesis & 1487 & 24 & 7.73 & 3.11 & 4.57E-03 \\
\hline Regulation of developmental process & 2263 & 30 & 11.76 & 2.55 & $8.48 \mathrm{E}-03$ \\
\hline Neuron differentiation & 937 & 18 & 4.87 & 3.7 & $1.38 \mathrm{E}-02$ \\
\hline Cell development & 1476 & 23 & 7.67 & 3 & $1.50 \mathrm{E}-02$ \\
\hline Regulation of biological quality & 3560 & 39 & 18.5 & 2.11 & $1.51 \mathrm{E}-02$ \\
\hline Regulation of multicellular organismal development & 1714 & 25 & 8.91 & 2.81 & $1.60 \mathrm{E}-02$ \\
\hline Anatomical structure morphogenesis & 1959 & 27 & 10.18 & 2.65 & $1.66 \mathrm{E}-02$ \\
\hline Single organism signaling & 5262 & 50 & 27.35 & 1.83 & $1.69 \mathrm{E}-02$ \\
\hline Signaling & 5266 & 50 & 27.37 & 1.83 & $1.73 \mathrm{E}-02$ \\
\hline Regulation of cell projection organization & 586 & 14 & 3.05 & 4.6 & $1.92 \mathrm{E}-02$ \\
\hline Single-organism cellular process & 9804 & 75 & 50.96 & 1.47 & 2.22E-02 \\
\hline Regulation of nervous system development & 782 & 16 & 4.06 & 3.94 & $2.52 \mathrm{E}-02$ \\
\hline Multicellular organismal signaling & 129 & 7 & 0.67 & 10.44 & 4.71E-02 \\
\hline
\end{tabular}

The top 189 transcripts, which had the highest number of human-specific intron motifs mapped to 135 UniProt IDs, which also mapped to 261 specific gene names. GO term analysis was performed with these genes at the Panther website. Shown below are the top 32 biological process $\mathrm{GO}$ terms found in this GO analysis

GC\% between any two modern human individuals can even exceed this level of variation. For example, Merchant et al. [47] estimated the GC\% of human to be $41 \%$, whereas we have $40.4 \%$. Furthermore, when we ran chi-squared analysis on the GC\% of the three human subspecies, we found that the $p$-values pertaining to each chi-square statistic were all statistically insignificant, given the null hypothesis that the GC\% of all three subspecies come from the same distribution. Therefore, we do not believe that these differences in GC\% between modern and archaic humans is statistically significant.
In order to get a picture of the biological differences between the motif distribution between the three genomes, we looked at the set of statistically significant motifs which were unique to modern human. We could only do this because gene annotation is available only for modern human, the other two subspecies being extinct. This was done separately for motifs of lengths $6-10$, for core, proximal, and distal promoters as well as introns between modern humans and Denisovan. This is because of the two archaic human subspecies, only Denisovan had these genomic subregions available. These 
Table 10 Cellular components found through GO term analysis

\begin{tabular}{|c|c|c|c|c|c|}
\hline GO term & Genes in database & Genes found & Expected & Fold enrichment & $P$-value \\
\hline Synapse & 793 & 21 & 4.12 & 5.1 & $1.05 \mathrm{E}-06$ \\
\hline Cell periphery & 5394 & 56 & 28.03 & 2 & $1.20 \mathrm{E}-05$ \\
\hline Cell junction & 1374 & 25 & 7.14 & 3.5 & 4.00E-05 \\
\hline Synapse part & 656 & 17 & 3.41 & 4.99 & 7.04E-05 \\
\hline Neuron part & 1310 & 24 & 6.81 & 3.52 & 7.04E-05 \\
\hline Plasma membrane & 5285 & 53 & 27.47 & 1.93 & $1.51 \mathrm{E}-04$ \\
\hline Cation channel complex & 207 & 10 & 1.08 & 9.29 & 1.97E-04 \\
\hline Ion channel complex & 283 & 11 & 1.47 & 7.48 & 3.93E-04 \\
\hline Transmembrane transporter complex & 321 & 11 & 1.67 & 6.59 & $1.34 \mathrm{E}-03$ \\
\hline Plasma membrane part & 2671 & 33 & 13.88 & 2.38 & $1.55 \mathrm{E}-03$ \\
\hline Transporter complex & 328 & 11 & 1.7 & 6.45 & $1.64 \mathrm{E}-03$ \\
\hline Postsynapse & 402 & 12 & 2.09 & 5.74 & $1.76 \mathrm{E}-03$ \\
\hline Neuron projection & 974 & 18 & 5.06 & 3.56 & $3.69 \mathrm{E}-03$ \\
\hline Cell projection & 1862 & 25 & 9.68 & 2.58 & $1.08 \mathrm{E}-02$ \\
\hline Potassium channel complex & 92 & 6 & 0.48 & 12.55 & $1.25 \mathrm{E}-02$ \\
\hline
\end{tabular}

Shown below are the top 15 cellular component GO terms found in the GO analysis at the Panther database

sets of statistically significant, modern human-unique motifs can be found in Additional file 9 (shown in the first four tabs for core, proximal and distal promoters). Also available in this file is the number of such motifs which were found in the top 100 (core, proximal, distal) promoter/intron region of the genes in modern human (Tables 5, 6, 7 and 8). In the "top genes (based on promoters) " tab of Additional file 9 we can see a list of 50 individual genes variants for core, proximal and distal promoters, which came from at least three of the top 100 lists mentioned previously.

These correspond to 38 individual genes, which are listed in Table 12, along with their annotation. The motif repertoire found in core promoters might be different from that of proximal and distal promoters because core promoter motifs take part in the active transcription of genes, whereas proximal/distal promoters play a more modulatory/regulatory role. This is because general transcription factors are found within the core promoters (therefore not too many genes were found with humanspecific motifs), whereas the proximal and distal promoters contain more specific regulatory motifs, unique to modern humans. When these 38 genes were entered into the GeneOntology database, two statistically significant (FDR $<5 \%$ ) GO terms came up: regulation of histone modification (GO:0031056), and animal organ development (GO:GO:0048513).

The human-unique intron motifs (lengths 6-10) were also searched for in the human intron sequence set, and the top 100 genes were selected with the highest number of human-unique motifs. These are also listed in Additional file 9. The tab "top genes (based on introns)" shows those 104 gene variants (corresponding to 59 genes), which were listed in at least three out of five top 100 gene lists. These 59 genes were searched for at the Gene Ontology website, and were shown to be associated with $33 \mathrm{GO}$ terms, listed in Table 13. What is interesting is that 11 of the $33 \mathrm{GO}$ terms were shown to be involved with neural activity: neuron projection development and morphogenesis, neuron development, neurogenesis, generation of neurons, neuron differentiation, nervous system development, and related terms, such as

Table 11 Number of significant genome motifs in nCONDEL and dCONDEL regions

\begin{tabular}{llllll}
\hline & Hexamers & Heptamers & Octamers & Nonamers & Decamers \\
\hline Neanderthal & 24 & 107 & 627 & 2206 & 6365 \\
Denisovan & 24 & 107 & 627 & 2195 & 6356 \\
difference & 0 & 0 & 6 & 29 & 73 \\
Only in Denisovan & 0 & 0 & 3 & 9 & 32 \\
Only in Neanderthal & 0 & 0 & 3 & 20 & 41 \\
\hline
\end{tabular}

Five hundred eighty-three hCONDEL regions present in chimpanzee were BLASTED against the Neanderthal and Denisovan genomes, for which there were 287 nCONDELs and dCONDELs. The number of motifs (length 6-10 bp) present in Neanderthal and Denisovan are given, as well as the number of motifs present in both species or unique to either Neanderthal or Denisovan 
Table 12 List of 38 human genes with a high number of human-unique motifs found in proximal and distal promoters

\begin{tabular}{|c|c|}
\hline Gene symbol & GeneCard Annotation \\
\hline ANKRD11 & Ankyrin Repeat Domain 11 \\
\hline ANO9 & Anoctamin 9 \\
\hline C1orf170 & PPARGC1 And ESRR Induced Regulator, Muscle 1 \\
\hline CKB & Creatine Kinase B \\
\hline CUX1 & Cut Like Homeobox 1 \\
\hline DVL1 & Dishevelled Segment Polarity Protein 1 \\
\hline EEF1D & Eukaryotic Translation Elongation Factor 1 Delta \\
\hline FGFRL1 & Fibroblast Growth Factor Receptor Like 1 \\
\hline GNAQ & G Protein Subunit Alpha Q \\
\hline IGF1R & Insulin Like Growth Factor 1 Receptor \\
\hline IGF2 & Insulin Like Growth Factor 2 \\
\hline ING2 & Inhibitor Of Growth Family Member 2 \\
\hline KIF1A & Kinesin Family Member 1A \\
\hline KLF16 & Kruppel Like Factor 16 \\
\hline LIMD2 & LIM Domain Containing 2 \\
\hline MTA1 & Metastasis Associated 1 \\
\hline NOC2L & NOC2 Like Nucleolar Associated Transcriptional Repressor \\
\hline NSD1 & Nuclear Receptor Binding SET Domain Protein 1 \\
\hline POLE & DNA Polymerase Epsilon, Catalytic Subunit \\
\hline PQLC1 & PQ Loop Repeat Containing 1 \\
\hline PWWP2B & PWWP Domain Containing 2B \\
\hline RAC3 & Rac Family Small GTPase 3 \\
\hline RASA3 & RAS P21 Protein Activator 3 \\
\hline RFNG & RFNG O-Fucosylpeptide 3-Beta-N-Acetylglucosaminyltransferase \\
\hline RUNX1 & Runt Related Transcription Factor 1 \\
\hline SAMD11 & Sterile Alpha Motif Domain Containing 11 \\
\hline SAMD4A & Sterile Alpha Motif Domain Containing 4A \\
\hline SDC1 & Syndecan 1 \\
\hline SEMA4C & Semaphorin $4 C$ \\
\hline SKI & SKI Proto-Oncogene \\
\hline SLC19A1 & Solute Carrier Family 19 Member 1 \\
\hline SLC2A4RG & SLC2A4 Regulator \\
\hline SPHK1 & Sphingosine Kinase 1 \\
\hline STK11 & Serine/Threonine Kinase 11 \\
\hline SUV42OH1 & Lysine Methyltransferase 5B \\
\hline TRIM8 & Tripartite Motif Containing 8 \\
\hline TSEN54 & TRNA Splicing Endonuclease Subunit 54 \\
\hline UBTF & Upstream Binding Transcription Factor, RNA Polymerase I \\
\hline
\end{tabular}

Statistically significant human-specific motif lists (lengths 6-10) were determined for core, proximal and distal promoters. These motifs were searched for in the appropriate human promoter set. The top 100 genes with the highest number of such motifs in their promoters were listed. Those 38 genes listed below belonged to at least three top 100 lists, and were found to be in common between proximal and distal promoters

vocalization, sensory organ development, and neuromuscular processes controlling balance, and neuromuscular processes. GO terms for exon development and axonogenesis were also found. For cellular components, these
59 genes were associated with two other statistically significant GO terms, namely synapse (GO:0045202) and neuron part (GO:0097458). These results correlate well with what we found in the analysis of Human 
Table 13 GO terms (biological processes) found for the 59 genes with a high number of human-unique intron motifs

\begin{tabular}{|c|c|c|c|}
\hline GO term & GO term id & No. genes & FDR \\
\hline System development & GO:0048731 & 37 & $1.39 \mathrm{E}-08$ \\
\hline Multicellular organism development & GO:0007275 & 38 & 7.48E-08 \\
\hline Anatomical structure development & GO:0048856 & 39 & 9.04E-08 \\
\hline Developmental process & GO:0032502 & 40 & 1.06E-07 \\
\hline Multicellular organismal process & GO:0032501 & 43 & 3.78E-07 \\
\hline Nervous system development & GO:0007399 & 25 & 7.65E-07 \\
\hline Cell-cell adhesion via plasma-membrane adhesion molecules & GO:0098742 & 9 & 3.47E-05 \\
\hline Cell-cell adhesion & GO:0098609 & 11 & 8.49E-05 \\
\hline Animal organ development & GO:0048513 & 25 & 2.47E-04 \\
\hline Neuron differentiation & GO:0030182 & 14 & 3.44E-04 \\
\hline Homophilic cell adhesion via plasma membrane adhesion molecules & GO:0007156 & 7 & 5.16E-04 \\
\hline Biological adhesion & GO:0022610 & 13 & 9.89E-04 \\
\hline Cell adhesion & GO:0007155 & 13 & $1.01 \mathrm{E}-03$ \\
\hline Cellular developmental process & GO:0048869 & 26 & 1.19E-03 \\
\hline Neurogenesis & GO:0022008 & 16 & 2.13E-03 \\
\hline Cell differentiation & GO:0030154 & 25 & 2.70E-03 \\
\hline Generation of neurons & GO:0048699 & 15 & 4.06E-03 \\
\hline Anatomical structure morphogenesis & GO:0009653 & 18 & $5.49 \mathrm{E}-03$ \\
\hline Neuron development & GO:0048666 & 11 & 5.75E-03 \\
\hline Neuromuscular process & GO:0050905 & 5 & 1.04E-02 \\
\hline Sensory organ development & GO:0007423 & 9 & $1.10 \mathrm{E}-02$ \\
\hline Neuromuscular process controlling balance & GO:0050885 & 4 & 1.37E-02 \\
\hline Vocalization behavior & GO:0071625 & 3 & 2.08E-02 \\
\hline Cell projection morphogenesis & GO:0048858 & 8 & 2.63E-02 \\
\hline Neuron projection development & GO:0031175 & 9 & 2.64E-02 \\
\hline Cell development & GO:0048468 & 14 & 2.70E-02 \\
\hline Retina layer formation & GO:0010842 & 3 & 2.73E-02 \\
\hline Neuron projection morphogenesis & GO:0048812 & 8 & 2.76E-02 \\
\hline Plasma membrane bounded cell projection morphogenesis & GO:0120039 & 8 & 2.81E-02 \\
\hline Axonogenesis & GO:0007409 & 7 & 3.04E-02 \\
\hline Cell part morphogenesis & GO:0032990 & 8 & $3.22 \mathrm{E}-02$ \\
\hline Cell morphogenesis & GO:0000902 & 9 & 3.59E-02 \\
\hline Axon development & GO:0061564 & 7 & 4.66E-02 \\
\hline
\end{tabular}

Statistically significant human-specific motif lists (lengths 6-10) were determined for introns. These motifs were searched for in the human intron sequence set. The top 100 genes with the highest number of such motifs in their introns were listed. Those 59 genes were listed which belonged to at least 3 top 100 lists, and plugged into the Gene Ontology database. Below are listed $33 \mathrm{GO}$ terms associated with these 59 genes

Accelarated Regions, and even found that 11 of these top 59 genes match genes from the analysis of HAR regions (see Table 8). These genes found in modern human compared to Denisovan imply that the regulatory motifs found in them are responsible for differential development in neural functions, possibly cognitive abilities, as well as sensory perception and vocalization. These differences may have accrued after the divergence between modern and archaic humans.

Since the sequencing of the whole genome was made possible for Neanderthal and Denisovan [1-3], despite the degraded quality of DNA, it surely might be possible to sequence the genomes of such fossil hominin species such as Homo erectus, Homo habilis, or the newly discovered Homo naledi [48], for example. With the whole genome sequence of a larger number of fossil hominins available, we would be able to make large-scale genomic analyses and comparisons possible. Even though we do not have access to gene expression or protein data, we can still learn a lot from comparing the genome motif content between different modern and archaic human subspecies. 


\section{Additional files}

Additional file 1: List of statistically significant motifs for modern human. The motif sequence, number of observed and expected occurrences as well as motif score are listed for motif lengths 6-10 in the whole genome, core, proximal, and distal promoters, as well as all introns, $5^{\prime}$ and $3^{\prime}$ UTRS, as well as average score and standard deviation for all motif lengths and genomic sub-regions. (XLSX 219664 kb)

Additional file 2: List of statistically significant motifs for Neanderthal. The motif sequence, number of observed and expected occurrences as well as motif score are listed for motif lengths 6-10 in the whole genome, as well as average score and standard deviation for all motif lengths. (XLSX $43083 \mathrm{~kb}$ )

Additional file 3: List of statistically significant motifs for Denisovan. The motif sequence, number of observed and expected occurrences as well as motif score are listed for motif lengths 6-10 in the whole genome, core, proximal, and distal promoters, as well as all introns, as well as average score and standard deviation for all motif lengths and genomic sub-regions. (XLSX $191122 \mathrm{~kb}$ )

Additional file 4: List of statistically significant motifs for mouse. The motif sequence, number of observed and expected occurrences as well as motif score are listed for motif lengths 6-10 in the whole genome, core, proximal, and distal promoters, as well as all introns, 5' and 3' UTRS, as well as average score and standard deviation for all motif lengths and genomic sub-regions. (XLSX $247147 \mathrm{~kb}$ )

Additional file 5: $P$-values for common motifs between modern modern human and Denisovan. P-values were calculated for common motifs for the whole genome, core, proximal, and distal promoters as well as all introns according to the hypergeometric distribution for lengths 6-10 bp. The length of the motif, the number of possible motifs as well as the number of common motifs, as well as the $p$-value is listed. (PDF $319 \mathrm{~kb}$ )

Additional file 6: List of statistically significant motifs in 49 HAR regions studied by Pollard et al., 2006 [23]. List of statistically significant genomic motifs found in 26 HARs in modern human, Neanderthal and Denisovan and in 20 intronic HARs in modern human, Neanderthal and Denisovan, as well as a list of the 82 intronic motifs unique to modern humans. (XLSX $36 \mathrm{~kb}$ )

Additional file 7: Results of $\mathrm{GO}$ analysis. A list of 82 statistically significant intron motifs from 20 intron HARs unique to human is provided. The list of 135 Uniprot IDs is listed as well as the 261 human genes that they correspond to. The results of the GO analysis for biological processes and cellular components are listed. (XLSX $27 \mathrm{~kb}$ )

Additional file 8: Intergenic motifs in nCONDELs and dCONDELs. List of statistically significant motifs in nCONDELS and dCONDELs in Neanderthal and Denisovan. These are statistically significant whole genome motifs which were found in regions of the Neanderthal and Denisovan genomes which are missing from the genome of modern human. (XLSX $191 \mathrm{~kb}$ )

Additional file 9: List of human specific motifs for motif lengths 6-10 from core, proximal, distal promoters and introns as compared to Denisovan. Statistically significant motifs of lengths 6-10 from core, proximal, and distal promoters as well as introns unique to modern humans are listed (and not found in Denisovan). These motifs were found in the corresponding genomic subregion in modern human, and the number of such human-unique motifs were listed for each gene variant. From these lists the top 100 genes were selected which had the highest number of human-unique motifs in their promoter/intron. Listed in tab "top genes (based on promoters)" are genes which were found in at least three top 100 lists. This was done separately for core, proximal, and distal promoters, and also for introns in the tab "top genes (based on introns)". Fifty gene variants (38 genes) were found which both in the proximal and distal promoter sets appeared. One hundred four genes (their NP id, GeneCard name and annotation) were found which were listed in at least 3 top 100 gene lists whose introns contained statistically significant human-unique intron motifs. (XLSX $5181 \mathrm{~kb}$ )

\section{Abbreviations}

AMTN: Amelotin; ASIC: Acid-sensing ion channel; CAMTA1: Calmodulin-binding transcription activator 1 isoform 1; CENTG2: AGAP1 (ArfGAP with GTPase domain, ankyrin repeat and PH domain 1); CONDEL: CONserved DELetion; E2F1: E2F transcription factor 1; EGR1: Early growth response 1 factor; ENAM: Enamelin; EPD: European Promoter Database; ERV: Endogenous Retroviros; GRID1: Glutamate receptor ionotropic, delta-1 precursor; HACNS: Human accelerated conserved non-coding sequences; HAR: Human accelerated regions; HERV: Human Endogenous Retroviros; HND: Human, Neanderthal, Denisovan; KLF4: Kruppel-like factor 4; KLF5: Kruppel-like factor 5; MAPK: Mitogen activated kinase-like protein; NAV2: Neuron navigator 2; P53: Tumor protein 53; P63: Tumor protein 63; PFM: Position frequency matrix; PRDM16: PR domain containing 16 isoform 1; PWM: Position weight matrix; RREB: Ras Responsive Element Binding Protein; SorCS2: VPS10 domaincontaining receptor SorCS2 precursor; SP1: Sp1 transcription factor; SP2: Sp2 transcription factor; TRAPPC9: Trafficking protein particle complex 9; ZNF263: Zinc finger protein 263

\section{Acknowledgments}

The authors wish to thank Minjie Fan, University of Nebraska at Omaha, for his help with data analysis.

\section{Funding}

The Bioinformatics and Systems Biology Core has been supported by the Nebraska Research Initiative, UNMC, and the Nebraska INBRE

[2P20GM103427] and CCSG [5P30CA036727] awards.

\section{Availability of data and materials}

The datasets generated and/or analyzed during the current study are available at the following website: http://golgi.unmc.edu/ HumanMotifomeData/

\section{Authors' contributions}

MC designed the study, performed the analysis, and wrote the manuscript. MM, MF, LP, BW and MP generated the Neanderthal and Denisovan genome sequences. PX helped in statistical analysis. MP and CG helped in the study design and helped write the manuscript. All authors read and approved the final manuscript.

Ethics approval and consent to participate

No human or animal subjects were used during this study.

\section{Competing interests}

The corresponding author hereby declares that the authors have no competing interests.

\section{Publisher's Note}

Springer Nature remains neutral with regard to jurisdictional claims in published maps and institutional affiliations.

\section{Author details}

'Department of Genetics, Cell Biology and Anatomy, University of Nebraska Medical Center, College of Medicine, Omaha, NE 68198-5145, USA.

${ }^{2}$ Information Technology Services, University of Nebraska Medical Center, Omaha, NE 68198-5030, USA. ${ }^{3}$ School of Interdisciplinary Informatics, University of Nebraska at Omaha, Omaha, NE 68182-0116, USA. ${ }^{4}$ Buildertrend Solutions, Inc, Omaha, NE 68154, USA.

Received: 20 November 2017 Accepted: 22 April 2018

Published online: 18 June 2018

\section{References}

1. Green RE, Krause J, Briggs AW, Maricic T, Stenzel U, Kircher M, et al. A draft sequence of the Neanderthal genome. Science. 2010;328:710-22.

2. Meyer M, Kircher M, Gansauge MT, Li H, Racimo F, Mallick S, et al. A highcoverage genome sequence from an archaic Denisovan individual. Science. 2012;338:222-6.

3. Reich D, Green RE, Kircher M, Krause J, Patterson N, Durand EY, et al. Genetic history of an archaic hominin group from Denisovan cave in Siberia. Nature. 2010;468:1053-60.

4. Pennisi E. Human evolution. More genomes from Denisovan cave show mixing of early human groups. Science. 2013;340:799.

5. Carroll SB. Evo-devo and an expanding evolutionary synthesis: a genetic theory of morphological evolution. Cell. 2008;134:25-36. 
6. King MC, Wilson AC. Evolution at two levels in humans and chimpanzees. Science. 1975;188:107-16.

7. Prabhakar S, Visel A, Akiyama JA, Shoukry M, Lewis KD, Holt A, et al. Human-specific gain of function in a developmental enhancer. Science. 2008;321:1346-50

8. Frankel N, Erezyilmaz DF, McGregor AP, Wang S, Payre F, Stern DL. Morphological evolution caused by many subtle-effect substitutions in regulatory DNA. Nature. 2011;474:598-603.

9. Prabhakar S, Noonan JP, Pääbo S, Rubin EM. Accelerated evolution of conserved noncoding sequences in humans. Science. 2006;314:786.

10. Ding Q, Hu Y, Xu S, Wang CC, Li H, Zhang R, et al. Neanderthal origin of the haplotypes carrying the functional variant Val92Met in the MC1R in modern humans. Mol Biol Evol. 2014;31:1994-2003.

11. International Human Genome Sequencing Consortium. Initial sequencing and analysis of the human genome. Nature. 2001;409:860-921.

12. Filipowicz W, Bhattacharyya SN, Sonenberg N. Mechanisms of posttranscriptional regulation by microRNAs: are the answers in sight? Nat Rev Genet. 2008;9:102-14.

13. Cserháti M, Turóczy Z, Dudits D, Györgyey J. The rice word landscape-a detailed catalog of the rice motif content in the noncoding regions. OMICS. 2011;15:819-28.

14. Cserháti M. Motif content comparison between monocot and dicot species. Genomics Data. 2015:3:128-36.

15. Mathelier A, Zhao X, Zhang AW, Parcy F, Worsley-Hunt R, Arenillas DJ, et al. JASPAR 2014: an extensively expanded and updated open-access database of transcription factor binding profiles. Nucleic Acids Res. 2014;42:142-7.

16. Tempel S. Using and understanding RepeatMasker. Methods Mol Biol. 2012:859:29-51.

17. Dreos R, Ambrosini G, Cavin Périer R, Bucher P. EPD and EPDnew, highquality promoter resources in the next-generation sequencing era. Nucleic Acids Res. 2013;41:157-64.

18. Shao $X$, Shepelev $V$, Fedorov A. Bioinformatic analysis of exon repetition, exon scrambling and trans-splicing in humans. Bioinformatics. 2006;22:692-8.

19. Grillo G, Turi A, Licciulli F, Mignone F, Liuni S, Banfi S, et al. UTRdb and UTRsite (RELEASE 2010): a collection of sequences and regulatory motifs of the untranslated regions of eukaryotic mRNAs. Nucleic Acids Res. 2010;38:75-80.

20. Kozomara A, Griffiths-Jones S. miRBase: integrating microRNA annotation and deep-sequencing data. Nucleic Acids Res. 2011;39:152-7.

21. Geisler M, Kleczkowski LA, Karpinski S. A universal algorithm for genome-wide in silicio identification of biologically significant gene promoter putative cis-regulatory-elements; identification of new elements for reactive oxygen species and sucrose signaling in Arabidopsis. Plant J. 2006;45:384-98.

22. Lichtenberg J, Yilmaz A, Welch JD, Kurz K, Liang X, Drews F, et al. The word landscape of the non-coding segments of the Arabidopsis thaliana genome. BMC Genomics. 2009;10:463.

23. Pollard KS, Salama SR, Lambert N, Lambot MA, Coppens S, Pedersen JS, et al. An RNA gene expressed during cortical development evolved rapidly in humans. Nature. 2006;443:167-72.

24. Zhang G, Pei Z, Ball EV, Mort M, Kehrer-Sawatzki H, Cooper DN. Crosscomparison of the genome sequences from human, chimpanzee, Neanderthal and a Denisovan hominin identifies novel potentially compensated mutations. Hum Genomics. 2011;5:453-84.

25. Mi H, Huang $X$, Muruganujan A, Tang H, Mills $C$, Kang D, et al. PANTHER version 11: expanded annotation data from gene ontology and Reactome pathways, and data analysis tool enhancements. Nucleic Acids Res. 2017:45:183-9.

26. Cserháti M, Turóczy Z, Zombori Z, Cserzo M, Dudits D, Pongor S, et al. Prediction of new abiotic stress genes in Arabidopsis thaliana and Oryza sativa according to enumeration-based statistical analysis. Mol Gen Genomics. 2011:285:375-91.

27. Ghaleb AM, Nandan MO, Chanchevalap S, Dalton WB, Hisamuddin IM, Yang W. Krüppel-like factors 4 and 5: the yin and yang regulators of cellular proliferation. Cell Res. 2005;15:92-6.

28. Frietze $S$, Lan $X$, Jin VX, Farnham PJ. Genomic targets of the KRAB and SCAN domain-containing zinc finger protein 263. J Biol Chem. 2010;285(2):1393-403.

29. Thiagalingam A, De Bustros A, Borges M, Jasti R, Compton D, Diamond L, et al. RREB-1, a novel zinc finger protein, is involved in the differentiation response to Ras in human medullary thyroid carcinomas. Mol Cell Biol. 1996;16:5335-45.

30. Agoni L, Golden A, Guha C, Lenz J. Neandertal and Denisovan retroviruses. Curr Biol. 2012:22:437-8.
31. Lee A, Huntley D, Aiewsakun P, Kanda RK, Lynn C, Tristem M. Novel Denisovan and Neanderthal retroviruses. J Virol. 2014;88:12907-9.

32. Marchi E, Kanapin A, Byott M, Magiorkinis G, Belshaw R. Neanderthal and Denisovan retroviruses in modern humans. Curr Biol. 2013;23:994-5.

33. Brameier M. Genome-wide comparative analysis of microRNAs in three nonhuman primates. BMC Res Notes. 2010;3:64.

34. Lopez-Valenzuela M, Ramírez O, Rosas A, García-Vargas S, de la Rasilla M, Lalueza-Fox C, et al. An ancestral miR-1304 allele present in Neanderthals regulates genes involved in enamel formation and could explain dental differences with modern humans. Mol Biol Evol. 2012;29:1797-806.

35. Sire JY, Davit-Béal T, Delgado S, Gu X. The origin and evolution of enamel mineralization genes. Cells Tissues Organs. 2007;186:25-48.

36. McLean CY, Reno PL, Pollen AA, Bassan Al, Capellini TD, Guenther C, et al. Human-specific loss of regulatory DNA and the evolution of human-specific traits. Nature. 2011:471:216-9.

37. Marzinke MA, Mavencamp T, Duratinsky J, Clagett-Dame M. 14-3-3ع and NAV2 interact to regulate neurite outgrowth and axon elongation. Arch Biochem Biophys. 2013;540:94-100

38. Lane RF, St George-Hyslop P, Hempstead BL, Small SA, Strittmatter SM, Gandy S. Vps10 family proteins and the retromer complex in aging-related neurodegeneration and diabetes. J Neurosci. 2012;32:14080-6.

39. Glerup S, Bolcho U, Mølgaard S, Bøggild S, Vaegter CB, Smith AH, et al. SorCS2 is required for BDNF-dependent plasticity in the hippocampus. Mol Psychiatry. 2016;21:1740-51.

40. Mochida GH, Mahajnah M, Hill AD, Basel-Vanagaite L, Gleason D, Hill RS, et al. A truncating mutation of TRAPPC9 is associated with autosomal-recessive intellectual disability and postnatal microcephaly. Am J Hum Genet. 2009:85:897-902

41. Livide G, Patriarchi T, Amenduni M, Amabile S, Yasui D, Calcagno E, et al. GluD1 is a common altered player in neuronal differentiation from both MECP2mutated and CDKL5-mutated iPS cells. Eur J Hum Genet. 2015;23:195-201.

42. Inoue M, Iwai R, Tabata H, Konno D, Komabayashi-Suzuki M, Watanabe C, et al. Prdm 16 is crucial for progression of the multipolar phase during neural differentiation of the developing neocortex. Development. 2017;144:385-99.

43. Long C, Grueter CE, Song K, Qin S, Qi X, Kong YM, et al. Ataxia and Purkinje cell degeneration in mice lacking the CAMTA1 transcription factor. Proc Natl Acad Sci U S A. 2014:111:11521-6.

44. Cabo R, Alonso P, Viña E, Vázquez G, Gago A, Feito J, et al. ASIC2 is present in human mechanosensory neurons of the dorsal root ganglia and in mechanoreceptors of the glabrous skin. Histochem Cell Biol. 2015;143:267-76.

45. Perez CA, Ott J, Mays DJ, Pietenpol JA. p63 consensus DNA-binding site: identification, analysis and application into a p63MH algorithm. Oncogene. 2007:26:7363-70.

46. de Melo MB, Rodrigues IS, Akagi EM. Soares do Amaral N, Ling H, Monroig $P$, et al. MiR-223-5p works as an oncomiR in vulvar carcinoma by TP63 suppression. Oncotarget. 2016;7:49217-31.

47. Merchant SM, Prochnik SE, Vallon O, Harris EH, Karpowicz SJ, Witman GB et al. The Chlamydomonas genome reveals the evolution of key animal and plant functions. Science. 2007;318:245-50.

48. Berger LR, Hawks J, de Ruiter DJ, Churchill SE, Schmid P, Delezene LK, et al. Homo Naledi, a new species of the genus Homo from the Dinaledi chamber, South Africa. elife. 2015;10:4.

\section{Ready to submit your research? Choose BMC and benefit from}

- fast, convenient online submission

- thorough peer review by experienced researchers in your field

- rapid publication on acceptance

- support for research data, including large and complex data types

- gold Open Access which fosters wider collaboration and increased citations

- maximum visibility for your research: over $100 \mathrm{M}$ website views per year

At BMC, research is always in progress.

Learn more biomedcentral.com/submissions 